\title{
Exact solution of an Ising model with competing interactions on a Cayley tree
}

\author{
N N Ganikhodjaev ${ }^{1}$, C H Pah ${ }^{2}$ and M R B Wahiddin ${ }^{3}$ \\ ${ }^{1}$ Department of Mathematics, National University of Uzbekistan, Vuzgorodok 700095, Tashkent, \\ Uzbekistan \\ ${ }^{2}$ Institute of Mathematical Sciences, University of Malaya, 50603 Kuala Lumpur, Malaysia \\ ${ }^{3}$ Centre for Computational and Theoretical Sciences, Faculty of Science, International Islamic \\ University Malaysia, 53100 Kuala Lumpur, Malaysia
}

Received 14 November 2002, in final form 19 February 2003

Published 3 April 2003

Online at stacks.iop.org/JPhysA/36/4283

\begin{abstract}
The exact solution of an Ising model with competing restricted interactions on the Cayley tree, and in the absence of an external field is presented. A critical curve is defined where it is possible to get phase transitions above it, and a single Gibbs state is obtained elsewhere.
\end{abstract}

PACS number: $05.50 .+\mathrm{q}$

\section{Introduction}

We consider the semi-infinite Cayley tree $J^{2}$ of order 2, that is a graph having no cycles, from each vertex of which, except on vertex $x^{0}$, emanates exactly three edges and from vertex $x^{0}$, which is the root of the tree, emanates two edges (see figure 1).

The vertices $x$ and $y$ are called nearest neighbours, which is denoted by $\langle x, y\rangle$, if there exists an edge connecting them. The vertices $x$ and $y$ are called second neighbours, which is denoted by $\nmid x, y$, if there exists a vertex $z \in V$, such that $x, z$ and $y, z$ are nearest neighbours.

Let $V$ be the set of vertices of $J^{2}$. We use the notation

$$
W_{n}=\left\{x \in V: d\left(x^{0}, x\right)=n\right\}
$$

where $d\left(x^{0}, x\right)$ is the distance in $V$, that is, for any $x, y \in V, d(x, y)$ is the length of the shortest path $x_{0}=x, x_{1}, x_{2}, x_{d}=y$ in $V$ (here the pairs $\left\langle x_{0}, x_{1}\right\rangle, \ldots,\left\langle x_{d-1}, x_{d}\right\rangle$ are nearest neighbours); and

$$
V_{n}=\left\{x \in V: d\left(x^{0}, x\right) \leqslant n\right\}=\bigcup_{i=0}^{n} W_{i} .
$$

The root $x^{0}$ is called the 0th level and the vertices in $W_{n}$ are called the $n$th level. Let $W_{1}=\left\{x^{1}, x^{2}\right\}$ and $W_{2}=\left\{x^{3}, x^{4}, x^{5}, x^{6}\right\}$ (see figure 1) be the vertices of the first and second levels. 


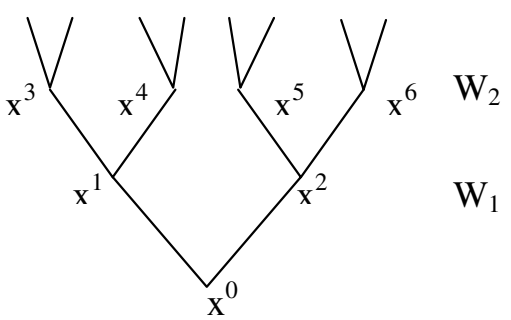

Figure 1. A semi-infinite Cayley tree $J^{2}$ of order 2, that is, a graph having no cycles, from each vertex of which, except vertex $x^{0}$, emanates exactly three edges and from vertex $x^{0}$, which is the root of the tree, emanates two edges.

The Ising model with competing interactions on the Cayley tree is defined by the Hamiltonian [1]

$$
H(\sigma)=-J \sum_{\langle x, y \backslash} \sigma(x) \sigma(y)-J_{1} \sum_{\langle x, y\rangle} \sigma(x) \sigma(y)-h \sum_{x \in V} \sigma(x)
$$

where the sum in the first term is taken over second neighbours, the sum in the second term is taken over nearest neighbours and the spin variables $\sigma(x)$ assume the values \pm 1 .

The problem of the phase transition for this particular model in such generality cannot be solved exactly [2-4]. In this paper we consider a slight modification to the Hamiltonian (1) and in this case we can exactly solve the resulting new model.

\section{The recurrent equations for partition functions}

There are several approaches to deriving the equation describing the limiting Gibbs measures for lattice models on the Cayley tree. One approach is based on properties of Markov random fields on Cayley tree [5-7]. Another approach is based on recurrent equations for partition functions [2]. Naturally both approaches lead to the same equation. However, the latter is more suitable for models with competing interactions.

Let $\Lambda$ be a finite subset of $V$. Assume that $\Omega(\Lambda)$ is the set of all configurations on $\Lambda$, that is the functions $\{\sigma(x), x \in \Lambda\}$. Let $\bar{\sigma}(V / \Lambda)$ be a fixed boundary configuration. The total energy of configuration $\sigma(\Lambda) \in \Omega(\Lambda)$ under condition $\bar{\sigma}(V / \Lambda)$ is defined as

$$
\begin{gathered}
H(\sigma(\Lambda) \mid \bar{\sigma}(V / \Lambda))=-J \sum_{\substack{|x, y\rangle \\
x, y \in \Lambda}} \sigma(x) \sigma(y)-J_{1} \sum_{\substack{\langle x, y\rangle \\
x, y \in \Lambda}} \sigma(x) \sigma(y)-h \sum_{x \in \Lambda} \sigma(x) \\
-J \sum_{\substack{|x, y\rangle \\
x \in \Lambda, y \notin \Lambda}} \sigma(x) \bar{\sigma}(y)-J_{1} \sum_{\substack{\langle x, y\rangle \\
x \in \Lambda, y \notin \Lambda}} \sigma(x) \bar{\sigma}(y) .
\end{gathered}
$$

When all boundary points $\{\bar{\sigma}(y), y \in V / \Lambda\}$ are fixed as 1 , we have the positive boundary and when they are fixed as -1 , we have negative boundary. The free boundary corresponds to the case when the last two sums above are absent, that is formally all boundary points are fixed as 0 .

The partition function $Z_{\Lambda}(\bar{\sigma}(V / \Lambda))$ in volume $\Lambda$ under boundary condition $\bar{\sigma}(V / \Lambda)$ is defined as

$$
Z_{\Lambda}=\sum_{\sigma(\Lambda) \in \Omega(\Lambda)} \exp (-\beta H(\sigma(\Lambda)) \mid \bar{\sigma}(V / \Lambda))
$$




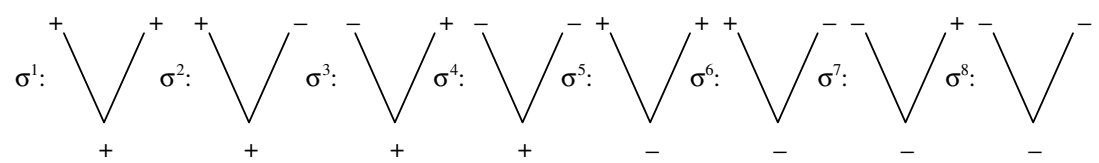

Figure 2. All configurations on $V_{1}=\left\{x^{0}, x^{1}, x^{2}\right\}$.

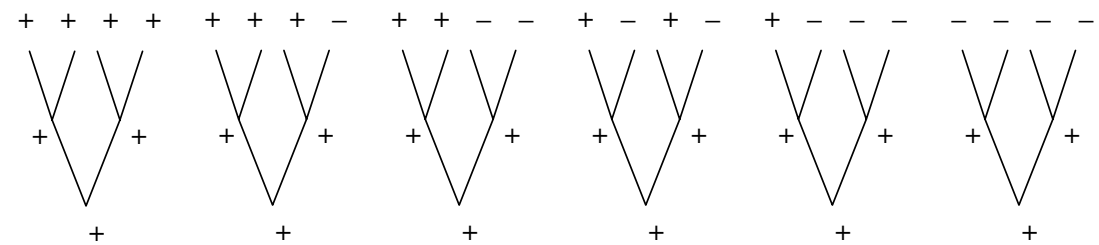

Figure 3. Six essentially different possibilities at the second level for $\sigma^{1}$.

where $\beta=\frac{1}{T}$ is the inverse temperature. Then the conditional Gibbs measure $\mu_{\Lambda}$ in volume $\Lambda$ under boundary condition $\bar{\sigma}(V / \Lambda)$ is defined as

$$
\mu_{\Lambda}(\sigma(\Lambda))=\frac{\exp (-\beta H(\sigma(\Lambda)) \mid \bar{\sigma}(V / \Lambda))}{Z_{\Lambda}} .
$$

We consider the configurations $\sigma\left(V_{n}\right)$, the partition functions $Z_{V_{n}}$ and conditional Gibbs measure $\mu_{\Lambda_{n}}$ in volume $V_{n}$ and for brevity we denote them as $\sigma_{n}, Z^{(n)}$ and $\mu_{n}$, respectively.

Consider the set of all configurations on $V_{1}=\left\{x^{0}, x^{1}, x^{2}\right\}$ (from figure 1) and enumerate them as shown in figure 2 .

We divide the partition function $Z^{(n)}$ into eight sums

$$
Z^{(n)}=\sum_{i=1}^{8} Z_{i}^{(n)}
$$

where

$$
Z_{i}^{(n)}=\sum_{\sigma_{n} \in \Omega\left(V_{n}\right):\left.\sigma_{n}\right|_{V_{1}}=\sigma^{i}} \exp \left(-\beta H_{n}\left(\sigma_{n}\right)\right) .
$$

Here, $\Omega\left(V_{n}\right)$ is the set of all configurations on $V_{n}$, and $H_{n}\left(\sigma_{n}\right)$ is the total energy of $\sigma_{n}$ with respect to either the positive boundary or the negative boundary or the free boundary.

Next, we consider the possibilities for the second level $W_{2}$ of our tree with $\sigma^{i}$ on the $V_{1}, i=1,2, \ldots, 8$. For example, there are six essentially different possibilities at the second level for $\sigma^{1}$ as shown in figure 3 .

We set

$$
\theta=\exp (\beta J) \quad \theta_{1}=\exp \left(2 \beta J_{1}\right) \quad \text { and } \quad \theta_{2}=\exp (\beta h) .
$$

It is not hard to deduce the following system of recurrent equations:

$$
\begin{aligned}
& Z_{1}^{(n)}=\theta \theta_{1} \theta_{2}\left[\theta^{2} Z_{1}^{(n-1)}+2 Z_{2}^{(n-1)}+\theta^{-2} Z_{4}^{(n-1)}\right]^{2} \\
& Z_{2}^{(n)}=Z_{3}^{(n)}=\theta^{-1} \theta_{2}\left[\theta^{2} Z_{1}^{(n-1)}+2 Z_{2}^{(n-1)}+\theta^{-2} Z_{4}^{(n-1)}\right]\left[\theta^{2} Z_{5}^{(n-1)}+2 Z_{6}^{(n-1)}+\theta^{-2} Z_{8}^{(n-1)}\right] \\
& Z_{4}^{(n)}=\theta \theta_{1}^{-1} \theta_{2}\left[\theta^{2} Z_{5}^{(n-1)}+2 Z_{6}^{(n-1)}+\theta^{-2} Z_{8}^{(n-1)}\right]^{2} \\
& Z_{5}^{(n)}=\theta \theta_{1}^{-1} \theta_{2}^{-1}\left[\theta^{-2} Z_{1}^{(n-1)}+2 Z_{2}^{(n-1)}+\theta^{2} Z_{4}^{(n-1)}\right]^{2} \\
& Z_{6}^{(n)}=Z_{7}^{(n)}=\theta^{-1} \theta_{2}^{-1}\left[\theta^{-2} Z_{1}^{(n-1)}+2 Z_{2}^{(n-1)}+\theta^{2} Z_{4}^{(n-1)}\right]\left[\theta^{-2} Z_{5}^{(n-1)}+2 Z_{6}^{(n-1)}+\theta^{2} Z_{8}^{(n-1)}\right] \\
& Z_{8}^{(n)}=\theta \theta_{1} \theta_{2}^{-1}\left[\theta^{-2} Z_{5}^{(n-1)}+2 Z_{6}^{(n-1)}+\theta^{2} Z_{8}^{(n-1)}\right]^{2} .
\end{aligned}
$$


We briefly describe how the first of these equations is obtained. According to figure 3 we have

$$
\begin{aligned}
Z_{1}^{(n)}=\theta \theta_{1} \theta_{2}[ & \theta^{4}\left(Z_{1}^{(n-1)}\right)^{2}+4 \theta^{2} Z_{1}^{(n-1)} Z_{2}^{(n-1)}+2 Z_{1}^{(n-1)} Z_{4}^{(n-1)} \\
+ & \left.4\left(Z_{2}^{(n-1)}\right)^{2}+4 \theta^{-2} Z_{2}^{(n-1)} Z_{4}^{(n-1)}+\theta^{-4}\left(Z_{4}^{(n-1)}\right)^{2}\right]
\end{aligned}
$$

where, for example, the coefficient of the first term in the square bracket is equal to $\theta^{4}$, because there are four pairs of second neighbours $\rangle x^{0}, x^{3}\langle,\rangle x^{0}, x^{4}\langle,\rangle x^{0}, x^{5}\langle$ and $\rangle x^{0}, x^{6}\langle$ the interactions of which are not considered in $Z_{1}^{(n-1)}$, and the coefficient $\theta_{2} \theta_{1} \theta$ in front of the square bracket is taken into account for the interactions on $\sigma^{1}$. It is evident that after some simplifications we obtain the first equation.

The full analysis of this system of recurrent equations is apparently difficult. In the next section we define a slight modification made to the Hamiltonian (1) and consequently system (2) is simplified.

\section{The one-level Ising model with competing interactions}

Definition 1. The second neighbours $>x, y<$ will be named one-level neighbours and are denoted by $\rangle x^{-}, y<$, if vertices $x$ and $y$ belong to $W_{n}$ for some $n$, that is if they are situated on the same level.

Definition 2. The one-level Ising model with competing interactions on the Cayley tree is defined by the Hamiltonian

$$
H(\sigma)=-J \sum_{\left\langle x^{-}, y\right\rangle} \sigma(x) \sigma(y)-J_{1} \sum_{\langle x, y\rangle} \sigma(x) \sigma(y)-h \sum_{x \in V} \sigma(x)
$$

where the sum in the first term, in contrast with the Hamiltonian (1), is only taken over one-level second neighbours.

We define

$$
Z_{+}^{(n)}\left(x^{0}\right)=Z_{1}^{(n)}+Z_{2}^{(n)}+Z_{3}^{(n)}+Z_{4}^{(n)}
$$

and

$$
Z_{-}^{(n)}\left(x^{0}\right)=Z_{5}^{(n)}+Z_{6}^{(n)}+Z_{7}^{(n)}+Z_{8}^{(n)}
$$

Then the system (2) is reduced to the following

$$
\begin{aligned}
& Z_{1}^{(n)}=\theta \theta_{1} \theta_{2}\left(Z_{+}^{(n-1)}\left(x^{0}\right)\right)^{2} \\
& Z_{2}^{(n)}=Z_{3}^{(n)}=\theta^{-1} \theta_{2}\left(Z_{+}^{(n-1)}\left(x^{0}\right)\right)\left(Z_{-}^{(n-1)}\left(x^{0}\right)\right) \\
& Z_{4}^{(n)}=\theta \theta_{1}^{-1} \theta_{2}\left(Z_{-}^{(n-1)}\left(x^{0}\right)\right)^{2} \\
& Z_{5}^{(n)}=\theta \theta_{1}^{-1} \theta_{2}^{-1}\left(Z_{+}^{(n-1)}\left(x^{0}\right)\right)^{2} \\
& Z_{6}^{(n)}=Z_{7}^{(n)}=\theta^{-1} \theta_{2}^{-1}\left(Z_{+}^{(n-1)}\left(x^{0}\right)\right)\left(Z_{-}^{(n-1)}\left(x^{0}\right)\right) \\
& Z_{8}^{(n)}=\theta \theta_{1} \theta_{2}^{-1}\left(Z_{-}^{(n-1)}\left(x^{0}\right)\right)^{2}
\end{aligned}
$$

since we do not take into account the interactions of second neighbours: $\rangle x^{0}, x^{3}\langle,\rangle x^{0}, x^{4}\langle$, $>x^{0}, x^{5}\langle$ and $\rangle x^{0}, x^{6}<$.

We define

$$
u_{n}\left(x^{0}\right)=\frac{Z_{+}^{n}\left(x^{0}\right)}{Z_{-}^{n}\left(x^{0}\right)} .
$$


Then, from system (4), by simple calculus we have

$$
u_{n}\left(x^{0}\right)=\theta_{2}^{2} \frac{\theta^{2} \theta_{1}^{2} u_{n-1}^{2}\left(x^{0}\right)+2 \theta u_{n-1}\left(x^{0}\right)+\theta^{2}}{\theta^{2} \theta_{1}^{2}+2 \theta_{1} u_{n-1}\left(x^{0}\right)+\theta^{2} u_{n-1}^{2}\left(x^{0}\right)} \quad n=2,3, \ldots
$$

Evidently,

$$
u_{n}\left(x^{0}\right)=\frac{\mu_{n}\left(\sigma_{n}\left(x^{0}\right)=1\right)}{\mu_{n}\left(\sigma_{n}\left(x^{0}\right)=-1\right)} .
$$

If we can find the limit of $u_{n}\left(x^{0}\right)$ as $n$ tends to infinity, we will find the ratio for the probability of a 1 to the probability of $\mathrm{a}-1$ at the root for the limiting Gibbs measure. Thus, the fixed points of the equation (6) describe the translation invariant limiting Gibbs measure of the model (3).

If $\theta=1$, that is $J=0$, equation (6) reduces to the well-known equation for the usual Ising model [2]

$$
u_{n}\left(x^{0}\right)=\theta_{2}^{2}\left(\frac{\theta u_{n-1}\left(x^{0}\right)+1}{u_{n-1}\left(x^{0}\right)+\theta_{1}}\right)^{2} \quad n=2,3, \ldots
$$

\section{The proof of existence of phase transitions}

We study the fixed points of equation (6) by solving the following equation:

$$
u=\theta_{2}^{2} \frac{\theta^{2} \theta_{1}^{2} u^{2}+2 \theta_{1} u+\theta^{2}}{\theta^{2} \theta_{1}^{2}+2 \theta_{1} u+\theta^{2} u^{2}} .
$$

In this paper we consider the case $h=0$, that is $\theta_{2}=1$, because in this situation we obtain a rather simple and descriptive answer concerning phase transitions. Our main result is the following:

Theorem. We consider a one-level Ising model with competing interactions (3), and with zero external field. Then, the curve $\theta^{*}=\frac{1+\sqrt{1+3 \theta^{4}}}{\theta^{2}}$ in the plane $\left(\theta, \theta_{1}\right)$ is a critical curve for phase transitions, namely, for an arbitrary pair of parameters $\left(\theta, \theta_{1}\right)$ above the critical curve a phase transition takes place and for any pair of parameters on or below the critical curve there occurs a single Gibbs state.

Proof. We consider the equation

$$
u=\frac{\theta^{2} \theta_{1}^{2} u^{2}+2 \theta_{1} u+\theta^{2}}{\theta^{2} \theta_{1}^{2}+2 \theta_{1} u+\theta^{2} u^{2}} .
$$

This equation is equivalent to the following cubic equation

$$
(u-1)\left(u^{2}+(\alpha+1) u+1\right)=0
$$

where

$$
\alpha=\frac{2 \theta_{1}-\theta_{1}^{2} \theta^{2}}{\theta^{2}}
$$

so that it is sufficient to investigate the quadratic equation

$$
u^{2}+(\alpha+1) u+1=0 .
$$

Equation (8) has two positive roots, if $\alpha<-3$. 


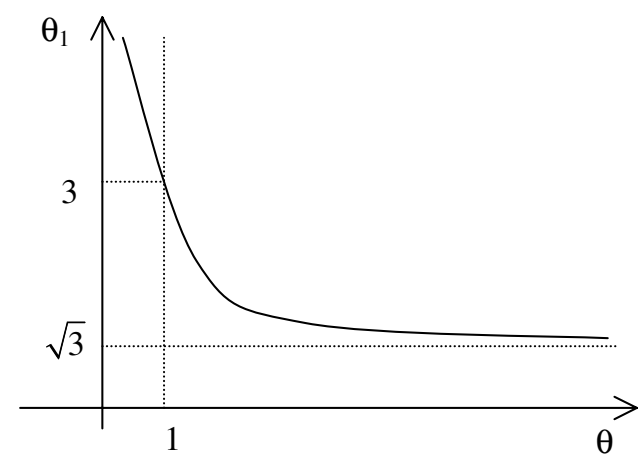

Figure 4. The curve $\theta^{*}=\frac{1+\sqrt{1+3 \theta^{4}}}{\theta^{2}}$ in the plane $\left(\theta, \theta_{1}\right)$.

This inequality is valid, if

$$
\theta_{1}>\frac{1+\sqrt{1+3 \theta^{4}}}{\theta^{2}} \text {. }
$$

Assume

$$
\theta^{*}=\frac{1+\sqrt{1+3 \theta^{4}}}{\theta^{2}} .
$$

The behaviour of this function has the following form (see figure 4). Thus for an arbitrary pair of parameters $\left(\theta, \theta_{1}\right)$, which is situated above the critical curve (9), equation (7) has three positive different roots, $u_{1}^{*}, u_{2}^{*}=1, u_{3}^{*}$ and for pairs which are situated on or below the critical curve (9), equation (7) has a single positive root $u^{*}=1$.

Note that if $\theta=1$, we have $\theta_{1}^{*}=3$. This critical value of $\theta_{1}$ is well known for the usual Ising model [4]. Now we return to equation (7) and set

$$
\psi(u)=\frac{\theta^{2} \theta_{1}^{2} u^{2}+2 \theta_{1} u+\theta^{2}}{\theta^{2} \theta_{1}^{2}+2 \theta_{1} u+\theta^{2} u^{2}} .
$$

It is not hard to show by simple calculus that in the domain above the critical curve (9) (see figure 4), this function will be increasing and will have a single positive point of inflection in the interval $\left(1, u_{3}^{*}\right)$, where $u_{3}^{*}$ is the largest fixed point.

It is easy to show diagrammatically (see figure 5) that if $0<u_{1}<u_{1}^{*}, u_{n}$ in equation (6) will monotonically increase to $u_{1}^{*}$, the smallest fixed point.

For values $u_{1}^{*}<u_{1}<u_{2}^{*}, u_{n}$ will decrease monotonically to $u_{1}^{*}$. For values $u_{2}^{*}<u_{1}<u_{3}^{*}$, $u_{n}$ will increase monotonically to $u_{3}^{*}$. Finally, if $u_{1}>u_{3}^{*}, u_{n}$ will decrease monotonically to $u_{3}^{*}$.

The smallest fixed point thus gives the limiting probability ratio for the positive boundary. The largest fixed point, on the other hand, gives the limiting probability ratio for the negative boundary, and the middle value $u_{2}^{*}=1$ corresponds to the free boundary.

Therefore, the roots $u_{1}^{*}$ and $u_{3}^{*}$ of equation (8) are stable fixed points of the recurrent equation (6). Hence, the theorem is proved.

It is necessary to note that if $\theta \geqslant 1$, which is the ferromagnetic case, one anticipates the existence of phase transitions. But for $\theta<1$, which is anti-ferromagnetic by $J$, an unexpected result is obtained.

The description of the Gibbs states corresponding to the roots $u_{1}^{*}, u_{2}^{*}=1, u_{3}^{*}$ will be given elsewhere. 


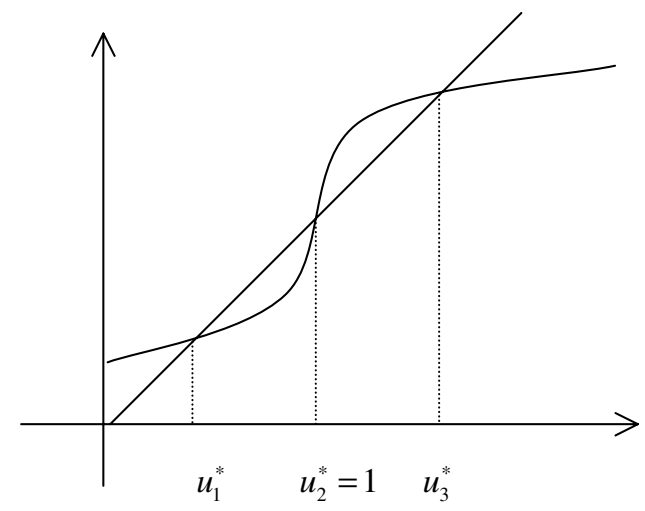

Figure 5. The largest stable fixed point $u_{3}^{*}$, the smallest stable fixed point $u_{1}^{*}$ and the middle value $u_{2}^{*}=1$ of the recurrent equation $(6)$.

\section{Conclusion}

The Ising model, which was originally regarded as a ferromagnetic model, has recently found some applications in many other physical, biological and chemical systems, and even in sociology. The model (1) considered above is a natural generalization of the Ising model, and a model of the form (3) has recently been investigated by Monroy [8, 9] to understand the physical aspects associated with the Husimi tree or the Kagome lattice. Interestingly, no exact solutions of the phase transition problem were found. Instead, some solutions for specific parameters were presented. On a similar note, the topic of statistical mechanics on nonamenable graphs is a modern growing field [10, 11].

We have presented the exact solution for the Hamiltonian (3). A critical curve is defined where it is possible to get phase transitions above it, and a single Gibbs state is found elsewhere.

\section{Acknowledgments}

This research is partly supported by a International Islamic University Malaysia short term research grant. One of us (NG) is indebted to the Institute of Mathematical Sciences, University of Malaya for a Visiting Professor appointment during the period 21 September 2000 to 20 March 2001. We are grateful to the referees, whose remarks facilitated the improvement of the paper.

\section{References}

[1] Katsura S and Takizawa M 1974 Prog. Theor. Phys. 51 82-98

[2] Kindermann R and Snell J L 1980 Markov random fields and their applications Contemp. Math. 199-112

[3] Mariz M, Tsallis C and Albuquerque E L 1985 J. Stat. Phys. 40 577-92

[4] da Silca CR and Coutinho S 1986 Phys. Rev. B 34 7975-85

[5] Georgii H O 1988 Gibbs measures and phase transitions De Gruyter Studies in Mathematics vol 9 (Berlin: de Gruyter)

[6] Spitzer F 1975 Ann. Prob. 3 387-98

[7] Preston K 1974 Gibbs States on Countable Sets (Cambridge: Cambridge University)

[8] Monroy J L 1992 J. Stat. Phys. 67 1185-2000

[9] Monroy J L 1994 Phys. Lett. A 188 80-4

[10] Lyons R 2000 J. Math. Phys. 41 1099-126

[11] Bleher P, Ruiz J, Schonmann R H, Schlosman S and Zagrebnov V 2001 Moscow Math. J. 1345-63 